\title{
Corticosteroid injection for adhesive capsulitis in primary care: a systematic review of randomised clinical trials
}

Kim Hwee Koh, MMed, FCFP

\begin{abstract}
Adhesive capsulitis is a common cause of shoulder pain and limited movement. The objectives of this review were to assess the efficacy and safety of corticosteroid injections for adhesive capsulitis and to evaluate the optimum dose and anatomical site of injections. PubMed and CENTRAL databases were searched for randomised trials and a total of ten trials were included. Results revealed that corticosteroid injection is superior to placebo and physiotherapy in the short-term (up to 12 weeks). There was no difference in outcomes between corticosteroid injection and oral nonsteroidal anti-inflammatory drugs at 24 weeks. Dosages of intra-articular triamcinolone $20 \mathrm{mg}$ and $40 \mathrm{mg}$ showed identical outcomes, while subacromial and glenohumeral corticosteroid injections had similar efficacy. The use of corticosteroid injections is also generally safe, with infrequent and minor side effects. Physicians may consider corticosteroid injection to treat adhesive capsulitis, especially in the early stages when pain is the predominant presentation.
\end{abstract}

Keywords: adhesive capsulitis, corticosteroids, frozen shoulder, injection

\section{INTRODUCTION}

Adhesive capsulitis or frozen shoulder is a common condition that presents with pain and progressive limitation of both active and passive shoulder movements. It is estimated to affect $2 \%-5 \%$ of the general population, and up to $20 \%$ of patients with diabetes mellitus. ${ }^{(1)}$ It can be either primary (idiopathic) or secondary; the latter includes causes such as rotator cuff tear, hemiparesis, cardiovascular diseases and diabetes mellitus. ${ }^{(1,2)}$

The American Shoulder and Elbow Surgeons (ASES) defines adhesive capsulitis as "a condition of uncertain aetiology characterised by significant restriction of both active and passive shoulder motion that occurs in the absence of a known intrinsic shoulder disorder ${ }^{\prime \prime}{ }^{(1,3)}$ However, there is no consensus on the exact range of motion (ROM) limitation of this condition, leading to confusion about its clinical diagnosis. ${ }^{(4)}$ Adhesive capsulitis is commonly described as passing through three stages: Stage 1 (freezing stage) with increasing pain and stiffness lasting 2-9 months; Stage 2 (frozen stage) with persistent stiffness lasting 4-12 months; and Stage 3 (thawing stage) with spontaneous recovery lasting 12-42 months. ${ }^{(5)}$ While commonly described as a self-limiting condition with spontaneous recovery within 2-3 years, up to $40 \%$ of patients may experience persistent symptoms, with $7 \%-15 \%$ having some degree of permanent functional loss. ${ }^{(4,6,7)}$

The goals of treatment are to relieve pain, restore movement and regain shoulder function. Common treatment options include nonsteroidal anti-inflammatory drugs (NSAIDs), corticosteroid injections and physical therapy, with more invasive treatments like capsular distension, manipulation under anaesthesia and arthroscopic capsular release being considered when conservative treatments fail. ${ }^{(6)}$ Most patients can be managed with nonoperative treatment in primary care. ${ }^{(6)}$ Intra-articular corticosteroid is widely used as a conservative treatment for adhesive capsulitis due to its cost-effectiveness and acceptance among patients. ${ }^{(8)}$ As adhesive capsulitis is postulated as an inflammatory and fibrotic disease, early treatment with intra-articular corticosteroid injections may reduce synovitis, limit the development of capsular fibrosis and alter the natural history of the disease..$^{(5,8,9)}$

Despite the multiple treatment options available for adhesive capsulitis, evidence for their efficacy is not well established, and it remains unclear whether it is better to use several interventions in combination. ${ }^{(4,6)}$ Previous systematic reviews on the use of corticosteroid in adhesive capsulitis mostly found evidence of its short-term effectiveness. ${ }^{(10-13)}$ In 2003, Buchbinder et $\mathrm{al}^{(10)}$ performed a systematic review of randomised and pseudorandomised trials on the use of corticosteroid injections (including 12 randomised controlled trials [RCTs] on adhesive capsulitis) in patients with shoulder pain; the authors concluded that, although the treatment may be beneficial, its effect may be small and not well maintained. In 2006, Shah and Lewis ${ }^{(11)}$ found that multiple (up to three) corticosteroid injections for the treatment of adhesive capsulitis improved pain and ROM for 6-16 weeks from the first injection. In 2009, Blanchard et al, ${ }^{(12)}$ who compared the effectiveness of corticosteroid injections with physiotherapeutic interventions for adhesive capsulitis, found greater improvement in pain, ROM and shoulder disability in favour of corticosteroid injections in the short-term (six weeks) and, to a lesser extent, in the longer-term (up to one year). Maund et al, in 2010, ${ }^{(13)}$ performed a systematic review and cost-effectiveness analysis on common interventions used in adhesive capsulitis and concluded that there may be short-term benefits from adding a single intraarticular steroid injection to home exercise for patients with primary frozen shoulder of less than six months' duration, but the evidence was limited.

With multiple new RCTs being performed in recent years, an updated review is required to evaluate both the short- and longterm efficacies of corticosteroid injections in adhesive capsulitis, as well as the optimum dose, anatomical site of injections and 
type of corticosteroids used. As corticosteroid injections are often used in combination with oral NSAIDs and physiotherapy for the treatment of adhesive capsulitis in primary care, a review comparing corticosteroid injections to these modalities would help to determine the best-available evidence, in order to inform the clinical decision-making process.

\section{METHODS}

\section{Literature search}

A literature search of electronic databases was performed on 26 June 2015. PubMed was searched from 1966 to the present, using the following search strategy: "adhesive capsulitis"[All Fields] OR "frozen shoulder" [All Fields]) AND (("Steroids" [MeSH] OR "steroid" [All Fields]) OR ("Glucocorticoids" [MeSH] OR "glucocorticoid" [All Fields]) OR ("Injections, IntraArticular" [MeSH] OR "injection"[All Fields])). CENTRAL was searched using a combination of the search terms: adhesive capsulitis, frozen shoulder, corticosteroid and injection. The limits were studies in the English language and those involving human studies. Only clinical trials were included in this review. The trials were initially selected on the basis of their titles and abstracts. The full text of articles that were deemed to be relevant to the review was retrieved and assessed. In addition, reference lists of shortlisted papers and other relevant systematic reviews were manually searched to identify additional studies that were not identified by our original search.

\section{Inclusion and exclusion criteria}

The inclusion criteria were randomised trials reporting efficacy on pain and/or function, and/or the safety of steroid injections versus placebo, oral NSAIDs or physiotherapy in patients with adhesive capsulitis. Those that compared different corticosteroid doses, types of corticosteroids and injection techniques, including intra-articular glenohumeral and subacromial injections, were also included. All image-guided techniques, including ultrasonography and fluoroscopy, were considered.

Excluded from the review were studies that: (a) investigated the treatment of shoulder pain from causes other than adhesive capsulitis; (b) investigated the treatment of secondary causes of adhesive capsulitis (except diabetes mellitus); (c) compared corticosteroid injection to oral corticosteroid, hyaluronate injection, hydrodilatation/distension arthrography or other surgical interventions such as manipulation under anaesthesia and arthroscopic capsular release; or (d) involved the use of corticosteroid injection in conjunction with distension arthrography and manipulation under anaesthesia (these treatment modalities are not commonly performed in primary care). In addition, studies that were assessed to be of low quality were excluded.

\section{Methodological assessment and data extraction}

Studies deemed eligible for inclusion were assessed for methodological quality using the Jadad scale. ${ }^{(14)}$ This contains two questions for randomisation and blinding, and one question for the reporting of withdrawals and dropouts. Each question entails a 'yes' or 'no' response option. In total, up to five points can be awarded, with higher scores indicating higher quality. Only studies deemed to be of high quality (Jadad score $\geq 3$ ) were included. In addition, as the Jadad scale does not contain allocation concealment, concealment of treatment allocation was assessed separately and scored as 'adequate', 'inadequate', or 'unclear' if there was insufficient information to make the judgement.

The following data was extracted from the studies: demographics (gender, mean age), duration of symptoms before treatment, comorbid diabetes mellitus, participants (eligibility criteria) and setting, interventions (site and number of injections, corticosteroid doses and volume, types of corticosteroid and image guidance), type of comparator, length of follow-up, assessment periods and outcomes. In order to evaluate safety, data on the number and type of adverse events reported was extracted from each study in the intervention and comparison groups.

\section{Outcome measures}

The primary outcome measures were pain as assessed by the visual analogue scale (VAS), ROM and shoulder function by any validated scale. Safety, a secondary outcome, was evaluated by the frequency of adverse events. Efficacy was assessed based on change in pain, ROM and functional scores, evaluated at baseline and the final assessment period. The clinical outcomes were then summarised in a narrative format due to heterogeneity in outcome measurements among the studies.

\section{RESULTS}

The initial database search identified 139 records and two additional records from the reference list check. There were 92 records after the removal of duplicates. 76 records were excluded after the titles and abstracts were reviewed for relevance, and 16 articles were eventually considered for inclusion. The fulltext manuscripts of the 16 studies were retrieved and reviewed. Of these, ten studies ${ }^{(8,9,15-22)}$ met the criteria for inclusion in the review. Six studies were excluded - five due to poor quality

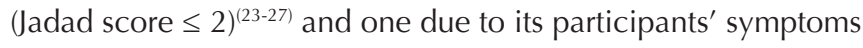
not being specific to adhesive capsulitis. ${ }^{(28)}$ The selection process is depicted in Fig. 1.

\section{Characteristics of included studies \\ Population}

Table I summarises the characteristics of the ten included studies. This review included 852 patients, of whom 445 (52.2\%) were women and $142(16.7 \%)$ were reported to have diabetes mellitus. The largest trial involved 191 patients $^{(21)}$ and the smallest included 45 patients. ${ }^{(8)}$ The mean age of the study participants was similar across all ten studies, with a maximum age of 60.2 years $^{(18)}$ and a minimum of 52.2 years. ${ }^{(9)}$ The mean pretreatment duration of symptoms in selected studies ranged from 12.2 weeks $^{(16)}$ to eight months. ${ }^{(20)}$ Only two studies reported the stage of adhesive capsulitis of the participants: the freezing and frozen stages in Roh et $\mathrm{al}^{\left({ }^{(8)}\right.}$ and freezing stage in Yoon et al. ${ }^{(9)}$ Seven of the studies were conducted in outpatient settings, ${ }^{(9,15-20)}$ of which one was 


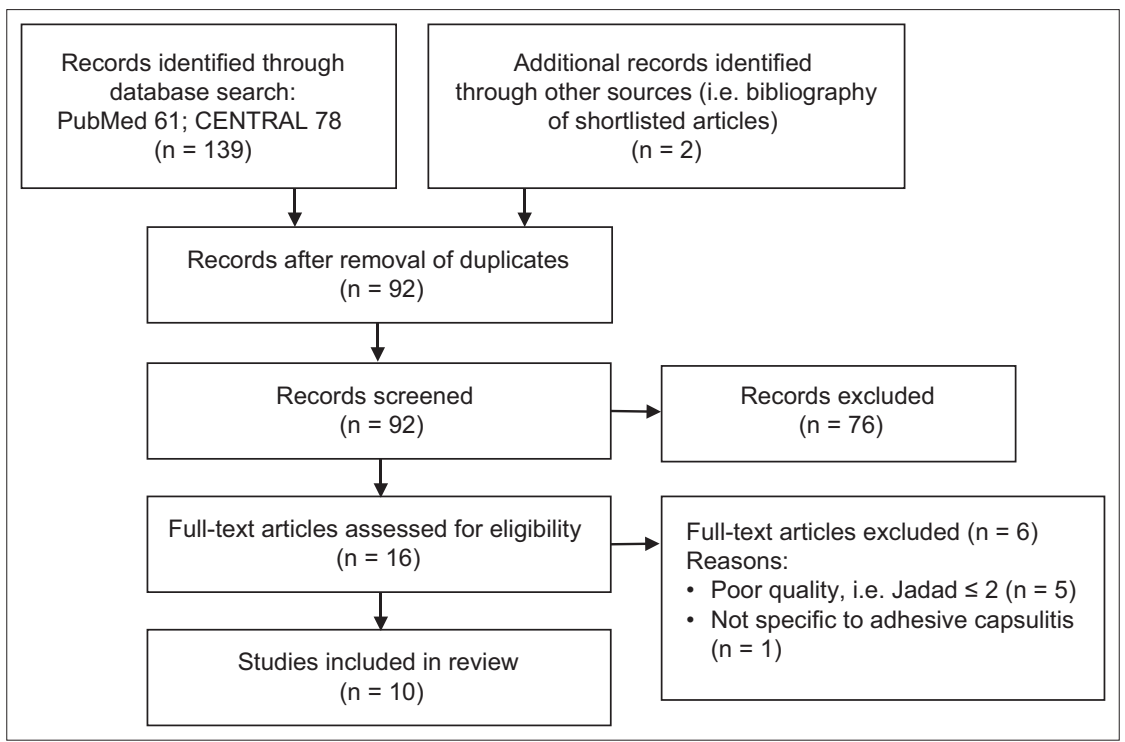

Fig. 1 Flow chart shows the study selection process.

conducted in general practice. ${ }^{(18)}$ There were differences in the inclusion criteria of the studies, particularly in the degree of loss of active and passive ROM.

\section{Interventions and comparison groups}

All the studies included corticosteroid injections in their interventions. Corticosteroid injection was compared with placebo/no injection in two studies, ${ }^{(8,15)}$ physiotherapy in three studies $^{(16-18)}$ and oral NSAIDs in one study. ${ }^{(19)}$ Two studies compared different doses of corticosteroids ${ }^{(9,20)}$ and another two studies compared different injection sites. ${ }^{(21,22)}$ None of the studies compared different types of corticosteroids.

The volumes and doses of corticosteroid, injection sites, image guidance used and number of injections were variable across all the studies. Triamcinolone was the most commonly used corticosteroid, ${ }^{(8,9,16-22)}$ and $40 \mathrm{mg}$ was the most frequently used dose..$^{(8,9,17-22)}$ Four studies ${ }^{(8,9,21,22)}$ had treatment groups that combined corticosteroid with lignocaine. The total volume of solution injected included $1 \mathrm{~mL},{ }^{(15)} 2 \mathrm{~mL},{ }^{(17)} 3 \mathrm{~mL},{ }^{(16)} 4 \mathrm{~mL},{ }^{(8)}$ $5 \mathrm{~mL}^{(9,21)}$ and $9 \mathrm{~mL} .^{(22)}$ Five studies used ultrasonography-guided injection $^{(8,9,19,21,22)}$ and one used fluoroscopic guidance. ${ }^{(17)}$ With the exception of two studies that compared glenohumeral and subacromial injections, ${ }^{(21,22)}$ seven studies used glenohumeral approaches $^{(8,9,15,17-20)}$ and one used a combined subacromialglenohumeral approach. ${ }^{(16)}$ Most studies used only a single corticosteroid injection, while two studies used multiple corticosteroid injections. ${ }^{(18,20)}$ Most of the studies utilised co-intervention in the form of home exercise programmes, but two studies completely avoided co-interventions. ${ }^{(18,20)}$ Most of the home exercises focused on passive ROM and pendulum exercises within the pain-free zone.

\section{Outcome measurements and assessment}

A number of outcome measures were utilised across the included studies. All the studies measured shoulder pain as an outcome using VAS scores, except for Carette et al, ${ }^{(17)}$ who used the
Shoulder Pain and Disability Index (SPADI) pain score. Active or passive ROM were measured in degrees using a goniometer or an inclinometer. ${ }^{(18)}$ For function and disability, several different clinical outcome measures were variably utilised (ConstantMurley, (22) SPADI, ${ }^{(9,15,17)}$ Shoulder Disability Questionnaire [SDQ] ${ }^{(16,18)}$ and ASES scores). ${ }^{(8,21)}$ Other outcome measures used included: the University of California-Los Angeles (UCLA) endresult score, ${ }^{(15)}$ which assessed the effectiveness of treatment; Short Form-36 (SF-36) Physical Component Summary and Mental Component Summary (MCS) scores $^{(17)}$ for quality of life assessment; patient satisfaction measured using VAS; ${ }^{(21)}$ and sleep disturbance using VAS. ${ }^{(20)}$

The final outcome assessment point of the included studies showed considerable variations, from six weeks after baseline ${ }^{(20)}$ to 52 weeks. ${ }^{(18)}$ Loss to follow-up was generally low, except for the studies by Bal et al $(n=16,20 \%),{ }^{(15)}$ Ryans et al ( $n=23$, $29 \%)^{(16)}$ and Dehghan et al $(n=18,24 \%) .{ }^{(19)}$

\section{Quality assessment}

Table II contains the Jadad and allocation concealment scores of the ten randomised clinical trials. There were variations in the methodological assessment scores in each of the included studies. A Jadad score of 3 was the minimum. ${ }^{(8,16-19,21,22)}$ Eight trials reported adequate concealment of allocation (80\%), $)^{(8,9,16-18,20-22)}$ only two trials were judged to have adequately blinded patients $(20 \%),{ }^{(9,15)}$ and seven had blinded outcome assessment (70\%). ${ }^{(9,15-18,21,22)}$ All ten studies accounted for all the participants at the end of the study.

\section{Clinical efficacy}

A summary of the clinical efficacy reported in the ten studies is shown in Table III.

\section{Intra-articular steroid injection versus placebo}

Two studies specifically examined steroid injection alone or compared it with a placebo injection. ${ }^{(8,15)}$ In Roh et al's study, ${ }^{(8)}$ 
Review Article

\section{Table I. Characteristics of included studies.}

\begin{tabular}{|c|c|c|c|c|c|c|c|}
\hline Study & Setting & Population & Intervention/comparison groups & Co-interventions & Injection technique & Outcome measures & Follow-up \\
\hline $\begin{array}{l}\text { Roh et al, } \\
2012^{(8)}\end{array}$ & $\begin{array}{l}\text { Actual site } \\
\text { NR, Seoul, } \\
\text { South Korea }\end{array}$ & $\begin{array}{l}n=45^{*} \\
\text { Inclusion criteria: DM patients with adhesive } \\
\text { capsulitis, shoulder pain with passive ROM } \\
\text { limitation }>25^{\circ} \text { or } 30^{\circ} \text { in at least } 2 \text { directions } \\
\text { compared to opposite shoulder or normal value, } \\
\text { unresponsive to stretching exercise with NSAIDs for } \\
3 \text { mth or if unable to participate in home stretching } \\
\text { exercise } \\
\text { Exclusion criteria: shoulder trauma, cerebrovascular } \\
\text { accident, endocrinal abnormality other than DM, } \\
\text { significant glenohumeral arthritis, blood coagulation } \\
\text { disorder, previous intra-articular therapy } \\
\text { Mean age: } 54.4-55.3 \text { yr } \\
\text { Mean symptom duration: } 6.2-6.5 \text { mth } \\
\text { Disease stage: freezing \& frozen } \\
\text { No. of DM patients included: } 45\end{array}$ & $\begin{array}{l}\text { Intra-articular } 40 \mathrm{mg}(1 \mathrm{~mL}) \\
\text { triamcinolone acetonide }+3 \mathrm{~mL} 2 \% \\
\text { lignocaine }(n=23) \\
\text { No injection }(n=22)\end{array}$ & $\begin{array}{l}\text { Home stretching } \\
\text { exercise + oral } \\
\text { NSAIDs }\end{array}$ & $\begin{array}{l}\text { USG intra-articular } \\
\text { injection, posterior } \\
\text { approach, single } \\
\text { injection }\end{array}$ & $\begin{array}{l}\text { Pain (10 cm VAS), } \\
\text { passive ROM } \\
\text { (measured with } \\
\text { goniometer), ASES } \\
\text { score }\end{array}$ & $\begin{array}{l}4,12 \\
24 w k\end{array}$ \\
\hline $\begin{array}{l}\text { Yoon } \\
\text { et al, } \\
2013^{(9)}\end{array}$ & $\begin{array}{l}\text { Outpatient, } \\
\text { Suwon, } \\
\text { Korea }\end{array}$ & $\begin{array}{l}n=53^{*} \\
\text { Inclusion criteria: Age } 20-70 \text { yr, primary adhesive } \\
\text { capsulitis, shoulder pain with restricted passive } \\
\text { ROM }>30^{\circ} \text { in } 2 \text { or more planes of movement, } \\
\text { symptom duration at least } 1 \mathrm{mth}, \\
\text { average pain } \geq 3 / 10 \text { on VAS } \\
\text { Exclusion criteria: secondary adhesive capsulitis, } \\
\text { rotator cuff lesions, previous corticosteroid } \\
\text { injections, use of antiplatelet or anticoagulant } \\
\text { Mean age: } 52.2-55.9 \mathrm{yr} \\
\text { Mean symptom duration: } 4.7-5.5 \mathrm{mth} \\
\text { Disease stage: freezing } \\
\text { No. of DM patients included: NR }\end{array}$ & $\begin{array}{l}\text { Intra-articular } 40 \mathrm{mg}(4 \mathrm{~mL}) \\
\text { triamcinolone acetonide }+1 \mathrm{~mL} 1 \% \\
\text { lignocaine }(n=20) \\
\text { Intra-articular } 20 \mathrm{mg}(2 \mathrm{~mL}) \\
\text { triamcinolone acetonide }+3 \mathrm{~mL} 1 \% \\
\text { lignocaine }(n=20) \\
\text { Intra-articular } 5 \mathrm{~mL} 1 \% \\
\text { lignocaine }(n=13)\end{array}$ & $\begin{array}{l}\text { Home exercise } \\
\text { programme } \times \\
12 \mathrm{wk}\end{array}$ & $\begin{array}{l}\text { USG intra-articular } \\
\text { injection, posterior } \\
\text { approach, single } \\
\text { injection }\end{array}$ & $\begin{array}{l}\text { SPADI score, global } \\
\text { shoulder pain } \\
\text { ( } 10 \mathrm{~cm} \text { VAS), passive } \\
\text { ROM (measured } \\
\text { with goniometer) }\end{array}$ & $\begin{array}{l}1,3,6 \\
12 w k\end{array}$ \\
\hline $\begin{array}{l}\text { Bal et al, } \\
2007^{(15)}\end{array}$ & $\begin{array}{l}\text { Outpatient, } \\
\text { Turkey }\end{array}$ & $\begin{array}{l}\mathrm{n}=80^{*} \\
\text { Inclusion criteria: } 18-70 \mathrm{yr} \text {, newly diagnosed } \\
\text { adhesive capsulitis, shoulder pain with limitation } \\
\text { of active and passive } \mathrm{ROM} \geq 25^{\circ} \text { in at least } \\
2 \text { directions, duration of symptom } 6 \text { wk to } 6 \mathrm{mth} \text {, no } \\
\text { prior treatment other than analgesia } \\
\text { Exclusion criteria: uncontrolled DM, } \\
\text { contraindications of injections, previous shoulder } \\
\text { surgery } \\
\text { Mean age: } 56.3-56.9 \mathrm{yr} \\
\text { Symptom duration: NR } \\
\text { Disease stage: NR } \\
\text { No. of DM patients included: NR }\end{array}$ & $\begin{array}{l}\text { Intra-articular } 40 \mathrm{mg}(1 \mathrm{~mL}) \\
\text { methylprednisolone acetate }(\mathrm{n}=40) \\
\text { Intra-articular } 1 \mathrm{~mL} 0.9 \% \text { normal saline } \\
(\mathrm{n}=40)\end{array}$ & $\begin{array}{l}\text { Home exercise } \\
\text { programme } \\
\times 12 \mathrm{wk} \text {, oral } \\
\text { paracetamol } \\
(1,500 \mathrm{mg} / \text { day }) \\
\text { when needed }\end{array}$ & $\begin{array}{l}\text { Blind intra-articular } \\
\text { injection, posterior } \\
\text { approach, single } \\
\text { injection }\end{array}$ & $\begin{array}{l}\text { SPADI score, } \\
\text { UCLA end-result } \\
\text { score, passive } \\
\text { ROM (measured } \\
\text { with goniometer), } \\
\text { night pain } \\
\text { (100 mm VAS) }\end{array}$ & $2,12 w k$ \\
\hline
\end{tabular}




\begin{tabular}{|c|c|c|c|c|c|c|c|}
\hline Study & Setting & Population & Intervention/comparison groups & Co-interventions & Injection technique & Outcome measures & Follow-up \\
\hline $\begin{array}{l}\text { Ryans } \\
\text { et al, } \\
2005^{(16)}\end{array}$ & $\begin{array}{l}\text { Outpatient, } \\
\text { UK }\end{array}$ & $\begin{array}{l}\mathrm{n}=78^{*} \\
\text { Inclusion criteria: Age }>18 \text { yr with diagnosis of } \\
\text { shoulder capsulitis, shoulder pain in C5 dermatome, } \\
\text { limitation of passive and active abduction and } \\
\text { external rotation }>25 \% \text { compared with opposite } \\
\text { shoulder, duration of symptom } 4 \text { wk to } 6 \text { mth } \\
\text { Exclusion criteria: pain < } 4 \text { wk or }>6 \text { mth, previous } \\
\text { intra-articular injection or physiotherapy, evidence } \\
\text { of glenohumeral osteoarthritis on X-ray, clinical } \\
\text { evidence of complete rotator cuff tear, significant } \\
\text { cervical spine disease, significant shoulder trauma, } \\
\text { inflammatory joint disease, cerebrovascular } \\
\text { accident, bilateral adhesive capsulitis, } \\
\text { contraindication to steroid injection } \\
\text { Mean age: } 52.3-56.3 \text { yr } \\
\text { Symptom duration: } 12.2-14.9 \text { wk } \\
\text { Disease stage: NR } \\
\text { No. of DM patients included: } 5\end{array}$ & $\begin{array}{l}\text { Injection and physiotherapy group: } \\
\text { Intra-articular } 20 \mathrm{mg}(1 \mathrm{~mL}) \\
\text { triamcinolone }+2 \mathrm{~mL} \text { normal saline } \\
+4 \text { wk physiotherapy } \\
\text { ( } \mathrm{n}=20 \text { ) } \\
\text { Injection-only group: Intra-articular } \\
20 \mathrm{mg}(1 \mathrm{~mL}) \text { triamcinolone }+2 \mathrm{~mL} \\
\text { normal saline only }(\mathrm{n}=19) \\
\text { Physiotherapy group: Intra-articular } \\
3 \mathrm{~mL} \text { normal saline injection }+4 \mathrm{wk} \\
\text { physiotherapy }(\mathrm{n}=20) \\
\text { Placebo group: } \\
\text { Intra-articular } 3 \mathrm{~mL} \text { normal saline } \\
\text { injection only }(\mathrm{n}=19)\end{array}$ & $\begin{array}{l}\text { Oral paracetamol, } \\
\text { home exercise } \\
\text { programme }\end{array}$ & $\begin{array}{l}\text { Blind combined } \\
\text { approach } \\
\text { (1.5 mL anterior } \\
\text { glenohumeral } \\
\text { injection and } \\
1.5 \mathrm{~mL} \text { lateral } \\
\text { subacromial } \\
\text { injection), single } \\
\text { injection }\end{array}$ & $\begin{array}{l}\text { Pain at rest } \\
\text { (100 mm VAS), } \\
\text { global improvement } \\
\text { (100 mm VAS), } \\
\text { passive external } \\
\text { rotation (measured } \\
\text { with goniometer), } \\
\text { SDQ score }\end{array}$ & $6,16 \mathrm{wk}$ \\
\hline $\begin{array}{l}\text { Carette } \\
\text { et al, } \\
2003^{(17)}\end{array}$ & $\begin{array}{l}\text { Outpatient, } \\
\text { Quebec \& } \\
\text { Ontario, } \\
\text { Canada }\end{array}$ & $\begin{array}{l}\mathrm{n}=93^{*} \\
\text { Inclusion criteria: Age }>18 \mathrm{yr} \text { with adhesive } \\
\text { capsulitis, shoulder pain with } \geq 25 \% \text { loss of active } \\
\text { and passive ROM in at least } 2 \text { directions compared } \\
\text { with contralateral shoulder, duration of symptom } \\
<1 \mathrm{yr}, \text { SPADI score } \geq 30 \\
\text { Exclusion criteria: secondary adhesive } \\
\text { capsulitis (except DM), known coagulation disorder, } \\
\text { contrast allergy } \\
\text { Mean age: } 54.2-56.5 \mathrm{yr} \\
\text { Mean symptom duration: } 20.3-22.1 \mathrm{wk} \\
\text { Disease stage: NR } \\
\text { No. of DM patients included: } 6\end{array}$ & $\begin{array}{l}\text { Injection and physiotherapy group: } \\
\text { Intra-articular } 40 \mathrm{mg}(2 \mathrm{~mL}) \\
\text { triamcinolone hexacetonide }+4 \mathrm{wk} \\
\text { physiotherapy }(\mathrm{n}=21) \\
\text { Injection-only group: Intra-articular } \\
40 \mathrm{mg}(2 \mathrm{~mL}) \text { triamcinolone } \\
\text { hexacetonide only }(\mathrm{n}=23) \\
\text { Physiotherapy group: Intra-articular } \\
2 \mathrm{~mL} \text { normal saline injection }+4 \mathrm{wk} \\
\text { physiotherapy ( } \mathrm{n}=26) \\
\text { Placebo group: Intra-articular } 2 \mathrm{~mL} \\
\text { normal saline injection only }(\mathrm{n}=23)\end{array}$ & $\begin{array}{l}\text { Home exercise } \\
\text { programme }\end{array}$ & $\begin{array}{l}\text { Fluoroscopic-guided } \\
\text { intra-articular } \\
\text { injection, single } \\
\text { injection }\end{array}$ & $\begin{array}{l}\text { SPADI, SF-36, } \\
\text { active and passive } \\
\text { ROM (measured } \\
\text { with goniometer) }\end{array}$ & $\begin{array}{l}6 \mathrm{wk}, 3 \\
6,12 \mathrm{mth}\end{array}$ \\
\hline $\begin{array}{l}\text { Van der } \\
\text { Windt } \\
\text { et al, } \\
1998^{(18)}\end{array}$ & $\begin{array}{l}\text { Outpatient, } \\
\text { general } \\
\text { practice, } \\
\text { Netherlands }\end{array}$ & $\begin{array}{l}n=109^{*} \\
\text { Inclusion criteria: Age }>18 \mathrm{yr} \text {, painful stiff shoulder } \\
\text { (capsular syndrome), painful and limited passive } \\
\text { ROM, with external rotation more restricted than } \\
\text { abduction and internal rotation } \\
\text { Exclusion criteria: bilateral symptoms, } \\
\text { corticosteroid injections or physiotherapy in } \\
\text { preceding } 6 \text { mth, contraindications to treatment, } \\
\text { shoulder surgery, dislocation or fracture, Type } 1 \\
\text { DM, systemic musculoskeletal or neurological } \\
\text { disorders } \\
\text { Mean age: } 57.3-60.2 \mathrm{yr} \\
\text { Symptom duration: }<1 \text { to }>12 \mathrm{mth} \\
\text { Disease stage: NR } \\
\text { No. of DM patients included: NR }\end{array}$ & $\begin{array}{l}\text { Intra-articular } 40 \text { mg triamcinolone } \\
\text { acetonide }(n=53) \\
\text { Physiotherapy: } 12 \text { sessions for } 6 \text { wk } \\
(n=56)\end{array}$ & $\begin{array}{l}\text { No } \\
\text { co-interventions }\end{array}$ & $\begin{array}{l}\text { Blind intra-articular } \\
\text { injection, posterior } \\
\text { approach; no more } \\
\text { than } 3 \text { injections } \\
\text { (average } 2.2 \\
\text { injections) in } 6 \mathrm{wk}\end{array}$ & $\begin{array}{l}\text { Pain associated with } \\
\text { main complaint, day } \\
\text { pain, night pain } \\
\text { (100 mm VAS), SDQ } \\
\text { score; overall clinical } \\
\text { severity (clinician } \\
\text { rated } 100 \text { mm VAS), } \\
\text { passive external } \\
\text { rotation and } \\
\text { abduction (measured } \\
\text { with inclinometer) }\end{array}$ & $\begin{array}{l}3,7,13, \\
26,52 \text { wk }\end{array}$ \\
\hline
\end{tabular}


Review Article

\begin{tabular}{|c|c|c|c|c|c|c|c|}
\hline Study & Setting & Population & Intervention/comparison groups & Co-interventions & Injection technique & Outcome measures & Follow-up \\
\hline $\begin{array}{l}\text { Dehghan } \\
\text { et al, } \\
2013^{(19)}\end{array}$ & $\begin{array}{l}\text { Outpatient, } \\
\text { Yazd, Iran }\end{array}$ & $\begin{array}{l}n=75^{*} \\
\text { Inclusion criteria: DM patients with adhesive } \\
\text { capsulitis, shoulder pain and limited shoulder ROM } \\
\text { in all directions } \\
\text { Exclusion criteria: symptoms }>6 \mathrm{mth} \text {, secondary } \\
\text { adhesive capsulitis, active peptic ulcer disease, } \\
\text { history of GI bleeding, coagulopathies, renal failure } \\
\text { Mean age: } 52.78-55.31 \text { yr } \\
\text { Symptom duration: NR } \\
\text { Disease stage: NR } \\
\text { No. of DM patients included: } 75\end{array}$ & $\begin{array}{l}\text { Intra-articular } 40 \mathrm{mg} \text { triamcinolone } \\
\text { acetonide }(n=40) \\
\text { Oral naproxen } 500 \mathrm{mg} B \text { for } \\
1 \mathrm{mth}(n=35)\end{array}$ & $\begin{array}{l}\text { Home exercise } \\
\text { programme }\end{array}$ & $\begin{array}{l}\text { USG intra-articular } \\
\text { injection, anterior } \\
\text { approach, single } \\
\text { injection }\end{array}$ & $\begin{array}{l}\text { Pain (10 cm VAS), } \\
\text { ROM (measured } \\
\text { with goniometer) }\end{array}$ & $\begin{array}{l}2,6,12, \\
24 \text { wk }\end{array}$ \\
\hline $\begin{array}{l}\text { Oh et al, } \\
2011^{(22)}\end{array}$ & $\begin{array}{l}\text { Actual site } \\
\text { NR, Seoul, } \\
\text { South Korea }\end{array}$ & $\begin{array}{l}n=71^{*} \\
\text { Inclusion criteria: primary frozen shoulder, shoulder } \\
\text { pain and limitation of active and passive ROM } \\
\text { in at least } 2 \text { directions, no improvement after } \\
\text { conservative management for at least } 6 \mathrm{wk} \\
\text { Exclusion criteria: secondary frozen shoulder } \\
\text { Mean age: } 55.7-58.3 \mathrm{yr} \\
\text { Mean symptom duration: } 6.2-6.9 \mathrm{mth} \\
\text { Disease stage: NR } \\
\text { No. of DM patients included: } 11\end{array}$ & $\begin{array}{l}40 \mathrm{mg}(1 \mathrm{~mL}) \text { triamcinolone }+4 \mathrm{~mL} \\
2 \% \text { lignocaine }+4 \mathrm{~mL} \text { normal saline into } \\
\text { glenohumeral joint }(n=37) \\
40 \mathrm{mg}(1 \mathrm{~mL}) \text { triamcinolone }+4 \mathrm{~mL} \\
2 \% \text { lignocaine }+4 \mathrm{~mL} \text { normal saline } \\
\text { into subacromial space }(n=34)\end{array}$ & $\begin{array}{l}\text { NSAIDs, analgesia, } \\
\text { home exercise } \\
\text { programme }\end{array}$ & $\begin{array}{l}\text { USG injection, } \\
\text { posterior or lateral } \\
\text { approach, single } \\
\text { injection }\end{array}$ & $\begin{array}{l}\text { Pain }(10 \mathrm{~cm} \\
\text { VAS), Constant } \\
\text { score, passive } \\
\text { ROM (measured } \\
\text { with goniometer) }\end{array}$ & $3,6,12 \mathrm{wk}$ \\
\hline
\end{tabular}

*No. of patients who were randomised. ASES: American Shoulder and Elbow Surgeons; BD: twice daily; DM: diabetes mellitus; NR: not recorded; NSAIDs: nonsteroidal anti-inflammatory drugs; ROM: range of motion; SDQ: Shoulder Disability Questionnaire; SPADI: Shoulder Pain and Disability Index; VAS: visual analogue scale; UCLA: University of California-Los Angeles; USG: ultrasonography-guided 
Table II. Quality assessment of included studies.

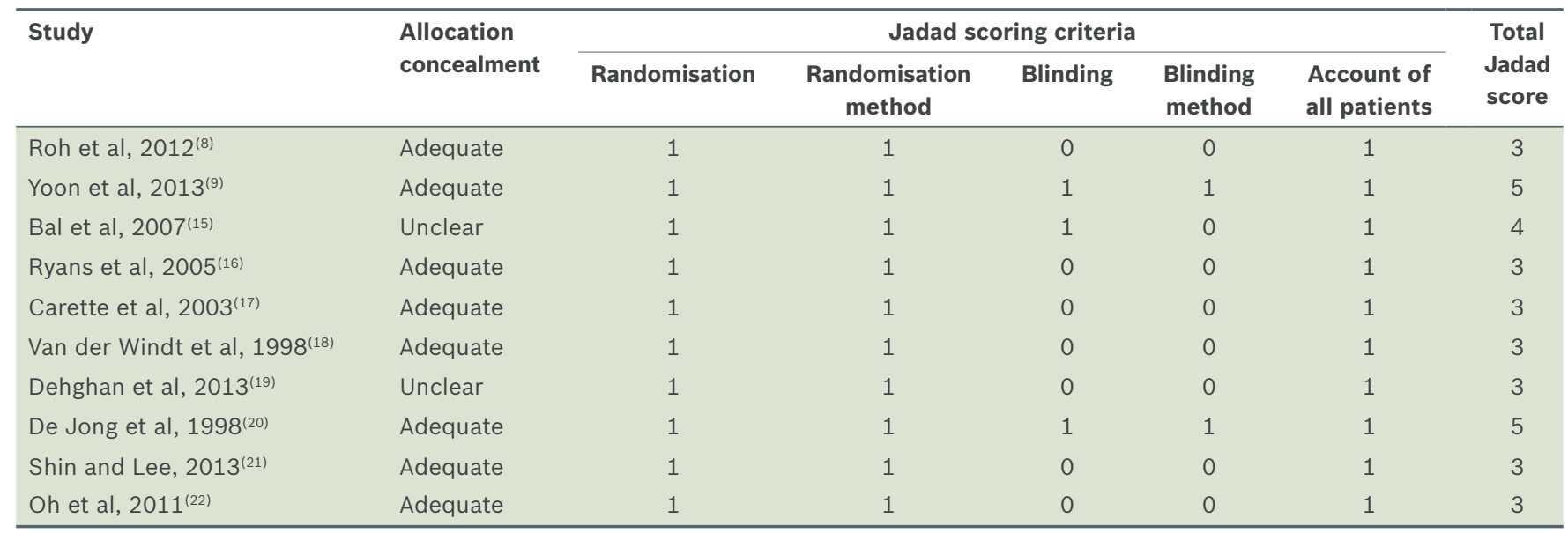

Table III. Clinical efficacy of the included studies.

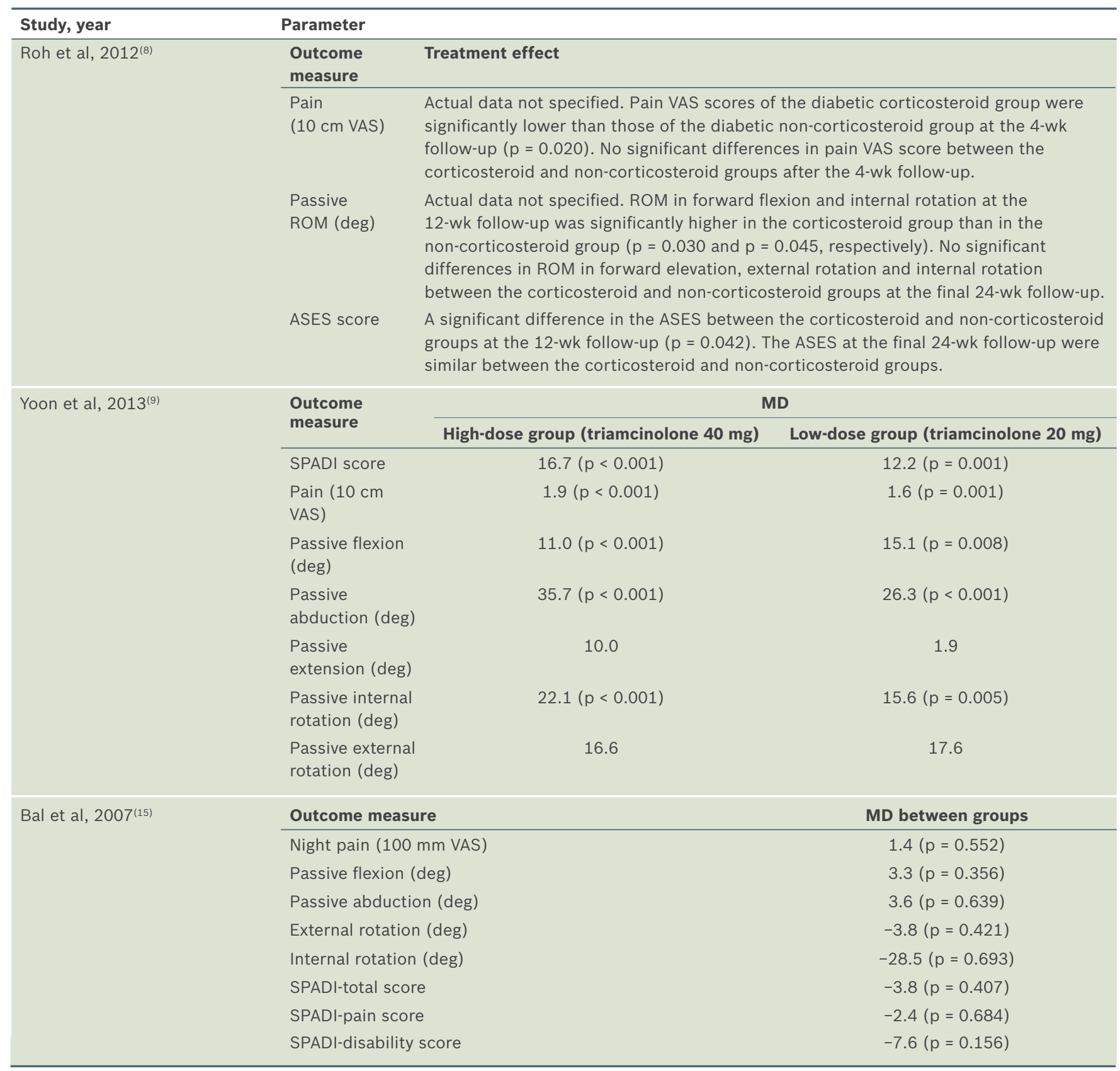




\begin{tabular}{|c|c|c|c|c|}
\hline Study, year & Parameter & & & \\
\hline \multirow[t]{6}{*}{ Ryans et al, $2005^{(16)}$} & \multirow{2}{*}{$\begin{array}{l}\text { Outcome } \\
\text { measure }\end{array}$} & \multicolumn{3}{|c|}{ MD $(95 \% \mathrm{Cl})$} \\
\hline & & $\begin{array}{l}\text { oup A (steroid injection } \\
\text { \& physiotherapy) }\end{array}$ & $\begin{array}{c}\text { Group B } \\
\text { (steroid injection only) }\end{array}$ & $\begin{array}{c}\text { Group C } \\
\text { (physiotherapy only) }\end{array}$ \\
\hline & SDQ score & $1.0(-3.4$ to 5.4$)$ & $1.3(-3.6$ to 6.1$)$ & $-1.0(-3.6$ to 6.1$)$ \\
\hline & VAS-global score & $-1.5(-21.7$ to 18.8$)$ & $-5.1(-27.8$ to 17.6$)$ & $2.0(-27.8$ to 17.6$)$ \\
\hline & $\begin{array}{l}\text { VAS-pain at rest } \\
\text { score }\end{array}$ & $-8.4(-30.7$ to 13.8$)$ & $-14.8(-40.0$ to 10.5$)$ & $4.8(-21.8$ to 31.5$)$ \\
\hline & $\begin{array}{l}\text { Passive external } \\
\text { rotation (deg) }\end{array}$ & $-2.5(-17.2$ to 12.3$)$ & $-3.1(-30.4$ to 27.9$)$ & $-4.2(-18.9$ to 12.7$)$ \\
\hline \multirow[t]{9}{*}{ Carette et al, $2003^{(17)}$} & \multirow{2}{*}{$\begin{array}{l}\text { Outcome } \\
\text { measure }\end{array}$} & \multicolumn{3}{|c|}{ MD $(95 \% \mathrm{Cl})$} \\
\hline & & $\begin{array}{c}\text { Group A (steroid } \\
\text { njection + physiotherapy) }\end{array}$ & $\begin{array}{l}\text { Group B (steroid } \\
\text { injection only) }\end{array}$ & $\begin{array}{c}\text { Group C (physiotherapy } \\
\text { only) }\end{array}$ \\
\hline & SPADI-total score & $-1.1(-15.5$ to 13.4$)$ & $-2.9(-17.0$ to 11.2$)$ & $1.7(-12.1$ to 15.4$)$ \\
\hline & SPADI-pain score & $-2.4(-18.4$ to 13.6$)$ & $-6.6(-22.2$ to 9.1$)$ & $-0.0(-15.3$ to 15.2$)$ \\
\hline & $\begin{array}{l}\text { SPADI-disability } \\
\text { score }\end{array}$ & $0.2(-13.8$ to 14.3$)$ & $0.8(-13.0$ to 14.6$)$ & $3.4(-10.0$ to 16.8$)$ \\
\hline & SF-36-PCS score & $1.4(-4.0$ to 6.7$)$ & $1.0(-4.3$ to 6.3$)$ & $-0.7(-5.9$ to 4.6$)$ \\
\hline & SF-36-MCS score & $6.1(-0.1$ to 12.3$)$ & $0.3(-5.8$ to 6.4$)$ & $-0.8(-6.9$ to 5.2$)$ \\
\hline & $\begin{array}{l}\text { Total active } \\
\text { ROM (deg) }\end{array}$ & $25.7(-0.6$ to 52.1$)$ & $0.0(-26.0$ to 26.0$)$ & $2.5(-22.8$ to 27.8$)$ \\
\hline & $\begin{array}{l}\text { Total passive } \\
\text { ROM (deg) }\end{array}$ & $19.6(-5.6$ to 44.8$)$ & $-6.7(-31.5$ to 18.2$)$ & $3.5(-20.7$ to 27.6$)$ \\
\hline \multirow[t]{5}{*}{ Van der Windt et al, $1998^{(18)}$} & \multicolumn{2}{|l|}{ Outcome measure } & \multicolumn{2}{|c|}{ MD $(95 \% \mathrm{Cl})$ between groups } \\
\hline & \multicolumn{2}{|c|}{ Pain associated with main complaint (100 mm VAS } & & 11 (1 to 23 ) \\
\hline & \multicolumn{2}{|l|}{ Day pain (100 mm VAS) } & & $3(-7$ to 13$)$ \\
\hline & \multicolumn{2}{|l|}{ Night pain (100 mm VAS) } & & $2(-12$ to 16$)$ \\
\hline & \multicolumn{2}{|l|}{$\mathrm{SDQ}$} & & $4(-10$ to 17$)$ \\
\hline \multirow[t]{6}{*}{ Dehghan et al, $2013^{(19)}$} & \multicolumn{2}{|l|}{ Outcome measure } & & MD between groups \\
\hline & \multicolumn{2}{|l|}{ Pain (10 cm VAS) } & & 0.26 \\
\hline & \multicolumn{2}{|l|}{ Flexion (deg) } & & 23.7 \\
\hline & \multicolumn{2}{|l|}{ Abduction (deg) } & & 11.5 \\
\hline & \multicolumn{2}{|l|}{ External rotation (deg) } & & -0.8 \\
\hline & \multicolumn{2}{|c|}{ Internal rotation (highest point above scapula) } & & -0.02 \\
\hline \multirow[t]{5}{*}{ De Jong et al, $1998^{(20)}$} & \multicolumn{2}{|l|}{ Outcome measure } & & MD between groups \\
\hline & \multicolumn{2}{|l|}{ Pain (100 cm VAS) } & & $18.1(p<0.01)$ \\
\hline & \multicolumn{2}{|l|}{ Disturbance of sleep } & & -0.003 (NS) \\
\hline & \multicolumn{2}{|l|}{ Functional impairment } & & $0.54(p=0.03)$ \\
\hline & \multicolumn{2}{|l|}{ External rotation restriction } & & $0.5(p=0.04)$ \\
\hline \multirow[t]{5}{*}{ Shin and Lee, $2013^{(21)}$} & Outcome measure & Treatment effect & & \\
\hline & Pain (10 cm VAS) & $\begin{array}{l}\text { Actual data not specifie } \\
8 \text { and } 16 \text { wk for all cort } \\
\text { to the oral NSAID grour } \\
\text { between all groups at }\end{array}$ & $\begin{array}{l}\text {. There was significantly } \\
\text { costeroid injection groups } \\
\text { (Group IV) }(p<0.05) \text {. No } \\
4 \text { wk }(p=0.67) \text {. }\end{array}$ & $\begin{array}{l}\text { greater pain relief at } 2,4 \text {, } \\
\text { (Groups I-III) compared } \\
\text { significant differences }\end{array}$ \\
\hline & $\begin{array}{l}\text { Patient satisfaction } \\
(10 \mathrm{~cm} \text { VAS) }\end{array}$ & $\begin{array}{l}\text { Actual data not specifie } \\
\text { satisfaction at 2, } 4,8 \text { an } \\
\text { (Groups I-III) compared } \\
\text { significant differences b }\end{array}$ & $\begin{array}{l}\text { There was significant imp } \\
16 \text { wk for all corticosteroi } \\
\text { to the oral NSAID group (G } \\
\text { tween all groups at } 24 \text { wk }\end{array}$ & $\begin{array}{l}\text { rovement in patient } \\
d \text { injection groups } \\
\text { roup IV) }(p=0.022) \text {. No } \\
(p=0.07) \text {. }\end{array}$ \\
\hline & Active flexion (deg) & $\begin{array}{l}\text { Actual data not specifie } \\
\text { forward flexion at 2, 4, } \\
\text { groups (Group I-III) cor } \\
\text { No significant differenc }\end{array}$ & $\begin{array}{l}\text { There was significant im } \\
\text { and } 16 \text { wk for all corticos } \\
\text { pared to the oral NSAID g } \\
\text { s between all groups at } 24\end{array}$ & $\begin{array}{l}\text { provement in active } \\
\text { teroid injection } \\
\text { roup (Group IV) }(p<0.05) \text {. } \\
\text { wk }(p=0.117)\end{array}$ \\
\hline & $\begin{array}{l}\text { Active external rotation } \\
(\text { deg) and active internal } \\
\text { rotation }\end{array}$ & $\begin{array}{l}\text { Actual data not specifie } \\
\text { throughout the follow- }-\end{array}$ & $\begin{array}{l}\text { No significant differenc } \\
\text { evaluations. }\end{array}$ & es between all groups \\
\hline
\end{tabular}




\begin{tabular}{lll}
\hline Study, year & Parameter & \\
\hline Oh et al, 2011(22) & Outcome measure & Treatment effect \\
\cline { 2 - 3 } & Constant scores & $\begin{array}{l}\text { Actual data not specified. No significant differences between groups } \\
\text { at 3,6 and 12 wk. } \\
\text { Passive abduction (deg) }\end{array}$ \\
& Passive internal rotation & \\
\hline
\end{tabular}

ASES: American Shoulder and Elbow Surgeons; Cl: confidence interval; deg: degrees; MD: mean difference; NS: not significant; NSAID: nonsteroidal anti-inflammatory drug; ROM: range of motion; SDQ: Shoulder Disability Questionnaire; SF-36-MCS: short form-36-Mental Component Summary; SF-36-PCS: short form-36-Physical Component Summary; SPADI: Shoulder Pain and Disability Index; VAS: visual analogue scale

significant improvement in pain score at four weeks was observed in diabetic patients who received intra-articular steroid injections, as compared to those who did not receive any injection ( $p=0.020)$, although no significant difference was seen beyond four weeks. There were significant improvements in functional score (based on ASES) $(p=0.042)$, forward elevation $(p=0.030)$ and internal rotation $(p=0.045)$ in those who had corticosteroid injections at 12 weeks, but not at 24 weeks. The limitations of this study included a small sample size $(n=45)$ and an absence of outcome assessor blinding, which could have introduced observation bias. ${ }^{(8)}$

In the study by Bal et al, ${ }^{(15)}$ intra-articular corticosteroids with home exercise resulted in statistically significant improvements in abduction $(p=0.033)$, SPADI total score $(p=0.047)$ and SPADI pain score $(p=0.041)$ compared to placebo injections with home exercise at two weeks. The UCLA score was significantly better in the steroids group $(p=0.002)$ compared to the placebo group. However, at 12 weeks, there was no significant difference between the two groups for night pain, SPADI scores, passive ROM and UCLA score. The limitations of this study included an absence of power analysis, unclear allocation concealment and a high loss to follow-up rate of $20 \%$. $^{(15)}$

\section{Intra-articular steroid injection versus physiotherapy}

Three studies compared steroid injection with physiotherapy. ${ }^{(16-18)}$ In van der Windt et al's study, ${ }^{(18)}$ corticosteroid injection was compared to physiotherapy, with a follow-up period of 52 weeks. However, they did not include a placebo group. The study found significant differences for all outcomes in favour of corticosteroid injection at all follow-up sessions, with the exception of severity rating of the main complaint and abduction, in which smaller differences were observed between the groups at 26 and 52 weeks. The authors also demonstrated treatment success (defined as complete recovery or much improvement) at seven weeks in $77 \%$ of patients treated with corticosteroid injections, as compared with $46 \%$ of those treated with physiotherapy (difference between groups 31\%, 95\% confidence interval $[\mathrm{Cl}]$ $14 \%-48 \%)$. ${ }^{(18)}$

Ryans et al ${ }^{(16)}$ compared intra-articular corticosteroid injection plus physiotherapy to steroid injection, physiotherapy alone or placebo. Factorial analysis showed that, at six weeks, there was significant improvement in SDQ score $(p=0.004)$ and global VAS $(p=0.040)$ in favour of steroid injection, but not for pain at rest $(p=0.838)$ and ROM ( $p=0.092)$, as well as significant improvement in passive external rotation in favour of physiotherapy ( $p=0.020)$. At 16 weeks, no significant difference was observed across interventions for all outcomes. Also, no significant interactions between injection and physiotherapy were noted at both six weeks and 16 weeks for all outcomes. One limitation of this study was its high loss to follow-up rate (29\%). ${ }^{(16)}$

Carette et $\mathrm{al}^{(17)}$ also performed a similar study comparing fluoroscopic-guided intra-articular corticosteroid injection plus physiotherapy to steroid injection, physiotherapy or placebo alone; however, the follow-up period was longer (12 months). At six weeks, intra-articular corticosteroid produced significantly greater improvement in total SPADI scores (pain and disability) as compared with physical therapy alone or placebo injection alone $(p=0.0004)$, with those who had both injection and physiotherapy experiencing greater improvement than those who had injection alone; the difference was, however, not statistically significant. At three months, significant improvement was seen in total SPADI and pain scores for combination therapy, as opposed to physiotherapy alone and the placebo group. There was also significant improvement in total SPADI score for the steroid group compared to the placebo group, but no significant difference was observed between the steroid and physiotherapy groups. At six months, there was no significant difference in SPADI scores between the four treatment groups. The combination group showed significant improvement in SF-36-MCS as well as active and passive ROM, compared to the physiotherapy and placebo groups. At 12 months, no significant difference was observed in all outcomes among the four groups. A limitation of this study was that no power analysis was performed. ${ }^{(17)}$

\section{Intra-articular steroid injection versus NSAID}

Only one study ${ }^{(19)}$ specifically compared intra-articular corticosteroid injection with oral NSAIDs. In this study, both the steroid injection and NSAID groups showed significant improvement for pain and ROM (24 weeks vs. two weeks, $p=0.001$ ). At 24 weeks, there were no significant differences between intra-articular corticosteroid injection and oral NSAIDs for all outcomes. There were, however, a number of limitations in this study: unclear adequacy of allocation concealment; the absence of a placebo group for comparison; outcome assessors that were not blinded; and a high loss to follow-up rate $(24 \%){ }^{(19)}$

\section{Comparison of intra-articular steroid injection dosages}

Two studies by De Jong ${ }^{(20)}$ and Yoon et al ${ }^{(9)}$ compared the dosages of intra-articular steroids. De Jong ${ }^{(20)}$ compared intra-articular 
triamcinolone $40 \mathrm{mg}$ (high-dose group) with triamcinolone $10 \mathrm{mg}$ (low-dose group) in patients with adhesive capsulitis. The high-dose group showed significant improvements in pain relief $(p<0.01)$, function $(p=0.03)$ and $\operatorname{ROM}(p=0.04)$ at six weeks, as compared to the low-dose group. The drawbacks in this study were that no power analysis was conducted, no placebo group was used, and blinding of patients and outcome assessors was unclear.

Yoon et al's study ${ }^{(9)}$ compared intra-articular triamcinolone $40 \mathrm{mg}$ (high-dose group) and intra-articular triamcinolone $20 \mathrm{mg}$ (low-dose group) with a placebo group. At 12 weeks, both the high- and low-dose groups showed significant improvements compared to the placebo group, in terms of pain $(p<0.001)$, disability $(p<0.001)$ and passive ROM of flexion $(p<0.01$ and $p=0.08$, respectively), abduction $(p<0.001)$ and internal rotation $(p<0.001$ and $p=0.005$, respectively). There was no statistically significance difference between the high- and low-dose groups for all outcomes at 12 weeks.

\section{Comparison of intra-articular steroid injection locations}

Different corticosteroid injection sites in adhesive capsulitis were examined in two studies. ${ }^{(21,22)}$ Oh et al, ${ }^{(22)}$ who compared intraarticular glenohumeral corticosteroid injections to subacromial injections, found no significant differences between the two groups at three, six and 12 weeks for all outcome measures of pain, function (Constant score) and passive ROM, with the exception of significant improvement in VAS pain $(p=0.023)$ in favour of glenohumeral injections at three weeks. There was no power analysis performed and no placebo group was used in this study.

Shin and Lee ${ }^{(21)}$ attempted a similar study but included an additional group with combined glenohumeral and subacromial injections, as well as a control group (oral NSAID). There were significant improvements in ASES score, pain score, forward flexion and patient satisfaction of up to 16 weeks for all corticosteroid injection groups (Groups I-III) compared to the oral NSAID group (Group IV) ( $p<0.05)$. At 24 weeks, all four groups showed significant improvement in ASES score compared to baseline $(p<0.05)$, but no intergroup difference was observed $(p=0.651)$.

\section{Safety of intra-articular steroid injection}

Among the included studies, only five $\mathrm{e}^{(9,18,20-22)}$ provided suitable data to assess the safety of corticosteroid injections. The main side effects were pain after injection (29.8\%), facial flushing (12.3\%) and menstrual irregularities (10.1\%). The number needed to harm for steroid injection versus physiotherapy was - 11.4 for pain, 7.1 for facial flushing and 9.5 for menstrual irregularities. None of the studies reported tendon rupture. This is consistent with the review by Shah and Lewis, ${ }^{(11)}$ which found that increased pain after injection (10\%-44\%), facial flushing $(12.5 \%-20 \%)$, rash $(4 \%)$ and irregular menstrual bleeding $(10.5 \%)$ were among the most common adverse effects of corticosteroid injection.

\section{LIMITATIONS OF REVIEW}

Due to restrictions in the literature search, some studies could have been missed, which may lead to bias. Publication bias may have arisen, since unpublished studies, which tend to be negative studies, were not searched. This may have resulted in an overestimation of the beneficial effects of corticosteroid injections. Since the studies were only reviewed by this author, there may have been bias in the scoring methodology.

\section{DISCUSSION}

The findings of this review indicate that treatment of adhesive capsulitis with corticosteroid injection leads to greater improvement in shoulder pain and function for up to 12 weeks, although its efficacy beyond 12 weeks has not been demonstrated. The findings were similar for the use of corticosteroid injections in diabetic patients with adhesive capsulitis. While no long-term efficacy was demonstrated in this review, adhesive capsulitis is widely regarded as a selflimiting disease, which may account for the minimal long-term differences between the interventions.

The similar degree of improvement in pain, function and ROM seen in the placebo group ${ }^{(15-17)}$ at longer term follow-up supports the notion that adhesive capsulitis has a favourable natural history. However, it can also be hypothesised that the steroid or placebo injection provided therapeutic benefit by causing distension of the joint capsule; hence, any improvement may be due to the volume of the injection rather than the corticosteroid itself. A recent Cochrane review ${ }^{(29)}$ investigated the efficacy of distension arthrography and found a lack of reliable evidence to establish the effectiveness of this technique. In addition, all the studies that involved placebo injections used the same volume as the steroid injection. The results showed that those who were injected with steroid had more significant improvement than those who were injected with saline or lignocaine, thus suggesting that the steroid component rather than the volume of the injection was responsible for the improvement.

Compared with physiotherapy alone, corticosteroid injections may offer significantly greater improvements in SPADI score, ${ }^{(17)}$ SDQ score, ${ }^{(16,18)}$ and $\mathrm{ROM}^{(18)}$ by six weeks of follow-up, but show similar outcomes at longer term follow-up beyond 12-16 weeks. ${ }^{(17,18)}$ Combined corticosteroid injection and physiotherapy treatment may also result in greater improvements in SPADI score and ROM than either treatment alone. ${ }^{(17)}$ The use of physiotherapy alone is, however, of limited benefit. ${ }^{(17)}$

Ryans et $\mathrm{al}^{(16)}$ found physiotherapeutic interventions to be more effective than corticosteroid injections in improving ROM in the early stages, as compared to the studies by Carette and van der Windt. ${ }^{(17,18)}$ A possible explanation was that this study used specific proprioceptive neuromuscular facilitation (PNF) exercises in the physiotherapeutic group, whereas PNF was not used in the other two studies. Another study ${ }^{(30)}$ found that treatment with PNF mobilisation led to a significant increase in ROM in patients with reduced external rotation of the shoulder and impaired overhead reach due to shoulder pathology. This could explain why Ryans et al's study ${ }^{(16)}$ was the only one that found physiotherapeutic interventions more effective than corticosteroid injections at improving external rotation. 
Most reviews advocated the use of physiotherapy and home exercises as a first-line treatment in adhesive capsulitis, regardless of the stage of disease. ${ }^{(1)}$ However, the use of physiotherapy alone is not supported by higher level studies, although several lower level studies reported benefit. ${ }^{(4)}$ A Cochrane review ${ }^{(31}$ of physiotherapy for painful conditions of the shoulder found no evidence that physiotherapy alone is beneficial in adhesive capsulitis. This is consistent with our findings.

Oral NSAIDs for adhesive capsulitis have not been shown to improve pain or function when compared with a placebo, ${ }^{(5)}$ although they are often prescribed in early inflammatory phases of the disease to provide pain relief. In some studies, ${ }^{(21)}$ NSAIDs were also used in control groups but were not considered to alter the course of the disease. However, in the study by Deghan et al, ${ }^{(19)}$ a single intra-articular corticosteroid injection and oral NSAIDs course for one month both showed similar improvements in pain and ROM at 24 weeks in diabetic patients, although no placebo group was included. Given that diabetic patients may have comorbidities (such as nephropathy or hypertension) and considering the potential adverse effects of NSAIDs (such as gastrointestinal bleeding), steroid injections for adhesive capsulitis may be a better therapy of choice than prolonged NSAID use in primary care.

Based on the results of studies that compared high- and lowdose corticosteroid injections, triamcinolone acetonide $40 \mathrm{mg}$ appears to be more effective in achieving pain relief than a dose of $10 \mathrm{mg}{ }^{(20)}$ but has a comparable outcome as a 20-mg dose $\mathrm{e}^{(9)}$ at 12 weeks. Based on this, Yoon et $\mathrm{al}^{(9)}$ recommended the use of triamcinolone acetonide $20 \mathrm{mg}$ as an initial dose to minimise the potential for local and systematic complications.

This review also found similar outcomes for pain and function regardless of whether corticosteroid injections were given via the subacromial, glenohumeral or combined approach. This may be explained by histologic studies that showed that contracture of the coracohumeral ligament, which lies in the subacromial space, is a main lesion in adhesive capsulitis. ${ }^{(5)}$ A previous study also found that the accuracy of blind intra-articular glenohumeral injections by experienced orthopaedic surgeons was less than $30 \%$. ${ }^{(32)}$ Hence this review's finding may be of clinical value in primary care, given the evidence that glenohumeral and subacromial injections have similar efficacy; the former, however, is technically more difficult to perform when conducted blindly, as the glenohumeral joint lies deep within the shoulder.

Based on the pathophysiology of adhesive capsulitis, one would assume that corticosteroid injection would be most effective in the earlier inflammatory stages of the disease and not in the latter stages when fibrous contracture is more apparent. While only two studies reported the stage of adhesive capsulitis, with the mean pretreatment duration of symptoms in most studies ranging from 12.2 weeks $^{(16)}$ to eight months, ${ }^{(20)}$ it is likely that most included patients were in the freezing or frozen stage, providing evidence for improved outcomes when corticosteroids are administered in the early stages of adhesive capsulitis.

This review also found that steroid injections are generally well tolerated, and have infrequent and minor side effects.
Pain was more frequently experienced in physiotherapy groups compared to corticosteroid groups. This could be due to the fact that aggressive physical therapy may exacerbate pain. Pain and limitation of movement can also be severe in the early inflammatory stage of adhesive capsulitis. Thus, physiotherapy may be difficult if it is undertaken during this painful phase, because the pain prevents the exercises from being carried out. Despite the lack of evidence of its long-term efficacy, the use of corticosteroid injections for improvement in short-term pain and disability would be valuable for patients. Therefore, a combination of corticosteroid injections to reduce initial pain, followed by physiotherapeutic interventions (such as mobilisation and exercise) to restore ROM and function, may be advocated.

Many of the included studies used clinical methods of diagnosing adhesive capsulitis, with secondary adhesive capsulitis being excluded with radiography, ultrasonography and physical examination. This may result in missed diagnosis of other shoulder disorders, such as labral lesions or small rotator cuff tears, which would have affected the patient's response to treatment. Due to the lack of an unambiguous definition for frozen shoulder, there were also differences in selection criteria (e.g. differences in loss of ROM) for each study.

Another limitation is that the participants were not blinded to the treatment given in some of the included studies, which may have resulted in some bias. However, successful blinding of participants to fulfil a double-blind study is difficult to achieve in practice, especially when comparing injection therapy to physiotherapy. Most of the studies ${ }^{(8,9,15-17,19,21,22)}$ used cointervention in the form of home exercise programmes, and the difficulty of ensuring compliance to an exercise programme may also have affected the validity of the results.

As the follow-up period for patients in most of the studies was up to a maximum of 52 weeks, another question that remains unanswered is whether corticosteroid injections merely afforded symptom relief or truly influenced the course of the condition. In addition, the adverse effects of corticosteroid injections may have not been fully assessed, as most of the studies were of a small size and short duration, and therefore could not detect rare adverse effects.

While most of the studies were conducted in outpatient settings, only one was based in general practice; hence, we should exercise caution when generalising the findings of this review to primary care. Furthermore, injections were given under radiological guidance in some of the included studies, ${ }^{(8,9,17,19,21,22)}$ so it may not be appropriate to generalise these findings to normal clinical settings in primary care.

\section{CONCLUSION}

This review incorporates a clinical perspective by comparing corticosteroid injections with other common modalities and evaluating the optimum corticosteroid dose and anatomical site of injection. Corticosteroid injections appear to be a useful and effective treatment option in adhesive capsulitis, as they can at least provide good short-term symptom relief, although their 
long-term efficacy has not been demonstrated. Corticosteroid injections have an additive effect to physiotherapy and home exercise programmes, and thus, may be prescribed concurrently. Patients in the early stages of disease who predominantly have pain symptoms may consider corticosteroid injection early, in an attempt to quickly resolve the symptoms before undergoing physiotherapy or home exercises. In primary care, a single subacromial injection of triamcinolone acetonide 20-40 mg may be used in the absence of imaging guidance.

\section{Implications for future research}

The adoption of a uniform definition and staging of adhesive capsulitis, combined with a standard set of outcome measures, would greatly enhance the value and generalisability of future research. While Shah and Lewis ${ }^{(11)}$ reported beneficial results with multiple injections, no RCTs have compared single versus multiple injections in adhesive capsulitis; thus, future studies may consider looking into this area. Future research may also consider measuring improvement/remission as an outcome, as it may be a more important patient-oriented outcome than increase in ROM or pain improvement. A dichotomous result would also enable the number needed to treat to be calculated.

\section{REFERENCES}

1. Hsu JE, Anakwenze OA, Warrender WJ, Abboud JA. Current review of adhesive capsulitis. J Shoulder Elbow Surg 2011; 20:502-14.

2. Neviaser AS, Neviaser RJ. Adhesive capsulitis of the shoulder. J Am Acad Orthop Surg 2011; 19:536-42.

3. Zuckerman JD, Rokito A. Frozen shoulder: a consensus definition. J Shoulder Elbow Surg 2011; 20:322-5.

4. Robinson CM, Seah KT, Chee YH, Hindle P, Murray IR. Frozen shoulder. J Bone Joint Surg Br 2012; 94:1-9.

5. Dias R, Cutts S, Massoud S. Frozen shoulder. BMJ 2005; 331:1453-6.

6. Guyver PM, Bruce DJ, Rees JL. Frozen shoulder - a stiff problem that requires a flexible approach. Maturitas 2014; 78:11-6.

7. Hand C, Clipsham K, Rees JL, Carr AJ. Long-term outcome of frozen shoulder. J Shoulder Elbow Surg 2008; 17:231-6.

8. Roh YH, Yi SR, Noh JH, et al. Intra-articular corticosteroid injection in diabetic patients with adhesive capsulitis: a randomized controlled trial. Knee Surg Sports Traumatol Arthrosc 2012; 20:1947-52.

9. Yoon SH, Lee HY, Lee HJ, Kwack KS. Optimal dose of intra-articular corticosteroids for adhesive capsulitis: a randomized, triple-blind, placebocontrolled trial. Am J Sports Med 2013; 41:1133-9.

10. Buchbinder R, Green S, Youd JM. Corticosteroid injections for shoulder pain. Cochrane Database Syst Rev 2003; (1):CD004016.

11. Shah N, Lewis M. Shoulder adhesive capsulitis: systematic review of randomised trials using multiple corticosteroid injections. Br J Gen Pract 2007; 57:662-7.

12. Blanchard V, Barr S, Cerisola FL. The effectiveness of corticosteroid injections compared with physiotherapeutic interventions for adhesive capsulitis: a systematic review. Physiotherapy 2010; 96:95-107.

13. Maund E, Craig D, Suekarran S, et al. Management of frozen shoulder: a systematic review and cost-effectiveness analysis. Health Technol Assess 2012; 16:1-264.

14. Jadad AR, Moore RA, Carroll D, et al. Assessing the quality of reports of randomized clinical trials: is blinding necessary? Control Clin Trials 1996; 17:1-12.

15. Bal A, Eksioglu E, Gulec B, et al. Effectiveness of corticosteroid injection in adhesive capsulitis. Clin Rehabil 2008; 22:503-12.

16. Ryans I, Montgomery A, Galway R, Kernohan WG, McKane R. A randomized controlled trial of intra-articular triamcinolone and/or physiotherapy in shoulder capsulitis. Rheumatology (Oxford) 2005; 44:529-35.

17. Carette S, Moffet H, Tardif J, et al. Intraarticular corticosteroids, supervised physiotherapy, or a combination of the two in the treatment of adhesive capsulitis of the shoulder: a placebo-controlled trial. Arthritis Rheum 2003; 48:829-38.

18. van der Windt DA, Koes BW, Devillé W, et al. Effectiveness of corticosteroid injections versus physiotherapy for treatment of painful stiff shoulder in primary care: randomised trial. BMJ 1998; 317:1292-6.

19. Dehghan A, Pishgooei N, Salami MA, et al. Comparison between NSAID and intra-articular corticosteroid injection in frozen shoulder of diabetic patients; a randomized clinical trial. Exp Clin Endocrinol Diabetes 2013; 121:75-9.

20. de Jong BA, Dahmen R, Hogeweg JA, Marti RK. Intra-articular triamcinolone acetonide injection in patients with capsulitis of the shoulder: a comparative study of two dose regimens. Clin Rehabil 1998; 12:211-5.

21. Shin SJ, Lee SY. Efficacies of corticosteroid injection at different sites of the shoulder for the treatment of adhesive capsulitis. J Shoulder Elbow Surg 2013; 22:521-7.

22. Oh JH, Oh $\mathrm{CH}$, Choi JA, et al. Comparison of glenohumeral and subacromial steroid injection in primary frozen shoulder: a prospective, randomized short-term comparison study. J Shoulder Elbow Surg 2011; 20:1034-40.

23. Mobini M, Kashi Z, Bahar A, Yaghubi M. Comparison of corticosteroid injections, physiotherapy, and combination therapy in treatment of frozen shoulder. Pak J Med Sci 2012; 28:648-51.

24. Arslan S, Celiker R. Comparison of the efficacy of local corticosteroid injection and physical therapy for the treatment of adhesive capsulitis. Rheumatol Int 2001; 21:20-3.

25. Rizk TE, Pinals RS, Talaiver AS. Corticosteroid injections in adhesive capsulitis: investigation of their value and site. Arch Phys Med Rehabil 1991; 72:20-2.

26. Dacre JE, Beeney N, Scott DL. Injections and physiotherapy for the painful stiff shoulder. Ann Rheum Dis 1989; 48:322-5.

27. Bulgen DY, Binder AI, Hazleman BL, Dutton J, Roberts S. Frozen shoulder: prospective clinical study with an evaluation of three treatment regimens. Ann Rheum Dis 1984; 43:353-60.

28. Hollingworth GR, Ellis RM, Hattersley TS. Comparison of injection techniques for shoulder pain: results of a double blind, randomised study. Br Med J (Clin Res Ed) 1983; 287:1339-41.

29. Buchbinder R, Green S, Youd JM, Johnston RV, Cumpston M. Arthrographic distension for adhesive capsulitis (frozen shoulder). Cochrane Database Syst Rev 2008; (1):CD007005.

30. Godges JJ, Mattson-Bell M, Thorpe D, Shah D. The immediate effects of soft tissue mobilization with proprioceptive neuromuscular facilitation on glenohumeral external rotation and overhead reach. J Orthop Sports Phys Ther 2003; 33:713-8.

31. Green S, Buchbinder R, Hetrick S. Physiotherapy interventions for shoulder pain. Cochrane Database Syst Rev 2003; (2):CD004258.

32. Sethi PM, Kingston S, Elattrache N. Accuracy of anterior intra-articular injection of the glenohumeral joint. Arthroscopy 2005; 21:77-80. 